\title{
miR-590 regulates WT1 during proliferation of G401 cells
}

\author{
LIYI HONG ${ }^{*}$, XU ZHAO*, XUEJUN SHAO and HONG ZHU \\ Clinical Medical Laboratory, Children's Hospital of Soochow University, Suzhou, Jiangsu 215004, P.R. China
}

Received September 29, 2015; Accepted February 22, 2017

DOI: $10.3892 / \mathrm{mmr} .2017 .6561$

\begin{abstract}
Nephroblastoma (Wilms' tumor) is frequently associated with mortality in children. MicroRNAs (miRNAs) are important for tumor development serving as oncogenes or tumor suppressors. In the present study, miRNA-590 (miR-590) was identified to be upregulated in Wilms' tumor tissues compared with the normal adjacent tissues. Additionally, the levels of miR-590 were consistent with their clinical stage. Wilms' tumor 1 (WT1) was considered to be a tumor suppressor in certain tumor types, and it has been detected at low expression levels in various types of cancer with high cell proliferation and aggressive behavior. The expression levels of miR-590 were quantified using reverse transcription-quantitative polymerase chain reaction. Cell proliferation was measured using 5-ethynyl-20-deoxyuridine assays. The protein expression levels of WT1 were investigated by western blot analysis. To the best of our knowledge, the present study was the first to determine that WT1 was a target gene of miR-590 as miR-590 was able to negatively regulate WT1 expression level by binding to the specific target site within the 3'-untranslated region (3'-UTR) of WT1 in G401 cells. Additionally, overexpression of miR-590 promoted G401 cell proliferation which was consistent with the effect of small interfering RNA-WT1. Subsequently, the present study determined that the cell phenotype altered by miR-590 overexpression may be reversed by upregulation of WT1 in G401 cells. In conclusion, the observations indicated that miR-590 may function as an oncogene via targeting WT1 to induce G401 cell proliferation. These results may contribute to current understanding of the function of miR-590 in nephroblastoma.
\end{abstract}

Correspondence to: Professor Hong Zhu, Clinical Medical Laboratory, Children's Hospital of Soochow University, 92 Zhongnan Street, Suzhou, Jiangsu 215004, P.R. China

E-mail: zhjyk2015@sina.com

${ }^{*}$ Contributed equally

Key words: nephroblastoma, miR-590, Wilms' tumor 1, G401 cells

\section{Introduction}

Wilms' tumor is one of the leading types of cancer and has become the primary cause of cancer-associated mortality in pediatric oncology worldwide (1). Clear cell renal cell carcinoma (CCRCC) is the primary type of kidney cancer of which 75-85\% and $80 \%$ of patients are at stage III and IV at the time of diagnosis, respectively, whereas the mixture of CCRCC and granular cell carcinoma are the predominant types in nephroblastoma (2). A previous study indicated that renal cancer incidence in children has increased globally in the last 10 years and the mortality rate remains high (3). In order to improve the clinical outcome, the underlying molecular mechanisms of the pathogenesis and progression of nephroblastoma require elucidation.

MicroRNAs (miRNAs) are a family of $\sim 19-22 \mathrm{bp}$, non-coding RNAs that function by regulating gene expression via binding to target mRNA and inducing mRNA cleavage or translational inhibition. Previous studies have demonstrated that miRNAs have an important role in various biological processes, including development, cell differentiation, proliferation and apoptosis $(4,5)$. Additionally, abnormal miRNA expression patterns occur in various types of cancer, although not all malignancies $(4,5)$. There has been increasing interest in investigating the importance of miRNA (miR)-590 in cancer progression. Previous studies reported that miR-590 was upregulated in hepatocellular carcinoma cells and CCRCC $(6,7)$. A previous study identified that miR-590 was a novel regulator of polybromo 1 (PBRM1) in CCRCC (7); therefore, increases the proliferation and invasion abilities of epithelial cells. These previous findings may suggest that miR-590 functions as a tumor activator in various types of cancer, although the contrary results have been obtained in other types of cancer including lung cancer and T-cell acute lymphoblastic leukaemia $(8,9)$. Several target genes of miR-590 associated with various types of cancer have been identified: Programmed cell death 4, transforming growth factor $\beta$ receptor type II, and phosphatase and tensin homolog in human hepatocellular cancer, PBRM1 in renal cancer, and cell adhesion molecule L1-like (CHL1) in cervical cancer $(6-8,10)$. However, the effects of miR-590 in CCRCC remain to be elucidated.

The present study determined that the expression level of miR-590 was increased in nephroblastoma tissues and the levels were consistent with the clinical stage of the tissue, which indicated that miR-590 was involved in the pathogenesis of Wilms' tumor. miR-590 induced G401 cell proliferation through Wilms' tumor 1 (WT1) as upregulation of miR-590 expression had the same effect as siRNA-WT1. 


\section{Materials and methods}

Clinical samples. Clinical Wilms' tumor samples were collected from 65 patients (male:female, 34:31; age, 5 months-7 years old) who underwent positive debulking surgery in the Pediatric Surgery Department of the First Affiliated Hospital of Suzhou University (Suzhou, China) between March 2013 and March 2015. The diagnosed Wilms' tumor tissues were reviewed by an experienced pathologist, using histological slides, according to the 2007 guidelines of WHO classification (11). The present study contained 25 stage I-II, 20 stage III and 20 stage IV renal cancer samples. Additionally, normal kidney tissue samples were obtained from 20 patients (male:female, 12:8; age, 5 months- 6.5 years old) at the Emergency Department with Kidney Trauma at the First Affiliated Hospital of Suzhou University between March 2013 and March 2015. The present study was approved by the Ethics Committee of the Children's Hospital of Soochow University (Suzhou, China) and informed consent was obtained from the guardians of all patients.

Plasmid construction. 3'-untranslated region (3'-UTR) of human WT1 was amplified by polymerase chain reaction (PCR) and the PCR fragment was inserted into the downstream of luciferase coding sequence of pcDNA3.1-luciferase reporter plasmid (Invitrogen; Thermo Fisher Scientific, Inc., Waltham, MA, USA) between the restriction site of BamHI and EcoRI. For 3'-UTR mutation, the predicted miR-590 binding site was randomly scrambled by PCR-mediated site-specific mutation. For WT1 overexpression in G401 cells, the coding DNA sequence (CDS) of WT1 was cloned into pCDNA3.1. The vectors used in the present study were verified by sequencing, and the primers were as follows: Wild type 3'-UTR of WT1 forward (F) 5'-CGGGATCCCTGGGAGTGT CCTTAGTGT-3' and reverse (R) 5'-CGGAATTCAACCCCA TTCAACCACAG-3'; mutant 3'-UTR of WT1 F 5'-TGACAA CTACCATTAGGACTG-3' and R 5'-ACAGTCCTAATGGTAGTTGTC-3'; CDS of WT1 F 5'-CCAAGCTTGGCTTTG CTGCTGAGGAC-3' R 5'-GCTCTAGAACCTCGGGAA TGTTAGAC-3'.

Cell culture. G401 cells were purchased from the Cell Bank of the Chinese Academy of Sciences (Shanghai, China). G401 cells were cultured in Dulbecco's modified Eagle's medium (DMEM; Invitrogen; Thermo Fisher Scientific, Inc.) supplemented with $10 \%$ fetal bovine serum (FBS) at $37^{\circ} \mathrm{C}, 5 \% \mathrm{CO}_{2}$ and $100 \%$ humidity.

Cell transfection. G401 cells were seeded at a density of $5 \times 10^{6}$ cells/well $16 \mathrm{~h}$ before transfection using DMEM medium with $1 \%$ penicillin/streptomycin. When cells reached $60 \%$ confluence, they were transfected with miRNA mimic, si-RNA WT1 (cat. no. sc-36846; Santa Cruz Biotechnology, Inc., Dallas, TX, USA) or expression vectors using Lipofectamine 2000 (Invitrogen; Thermo Fisher Scientific, Inc.) according to the manufacturer's protocol. Briefly, $10 \mathrm{nM}$ miRNA mimic, $100 \mathrm{nM}$ si-RNA, $800 \mathrm{ng}$ plasmid and Lipofectamine 2000 were diluted in the serum-free medium respectively at room temperature for $5 \mathrm{~min}$. After $5 \mathrm{~min}$, the diluted miRNA mimic, si-RNA or plasmid was mixed with the diluted Lipofectamine 2000 and incubated for $20 \mathrm{~min}$ at room temperature. The mixture was added to cells uniformly at $30 \mu \mathrm{l} /$ well. Cells were cultured at $37^{\circ} \mathrm{C}, 5 \% \mathrm{CO}_{2}$ for $6 \mathrm{~h}$, the medium was changed with fresh DMEM supplemented with $10 \%$ FBS and $1 \%$ penicillin/streptomycin.

Total RNA preparation and reverse transcription-quantitative PCR (RT-qPCR) analysis. Total RNA extracted from G401 cells and renal samples with TRIzol reagent (Invitrogen; Thermo Fisher Scientific, Inc.) were synthesized by RevertAid First Strand cDNA Synthesis kit (Thermo Fisher Scientific, Inc.) using the specific miR-590 RT primer supplied from Guangzhou RiboBio Co., Ltd. (Guangzhou, China). The qPCR was performed with $20 \mathrm{ng}$ cDNA using $2 \mathrm{X}$ UltraSYBR Mixture kit (Takara Bio, Inc., Otsu, Japan) according to the manufacturer's protocol. U6 was used as a reference gene determined at the same time. The miRNA qPCR detection primers purchased from Guangzhou RiboBio Co., Ltd. were used for qPCR analysis for miR-590 and U6. qPCR was performed in triplicate for each experiment. The cycling conditions were as follows: Initial denaturation at $95^{\circ} \mathrm{C}$ for $1 \mathrm{~min}, 30$ cyles of denaturation at $94^{\circ} \mathrm{C}$ for $30 \mathrm{sec}$, annealing at $58^{\circ} \mathrm{C}$ for $30 \mathrm{sec}$ and extension at $72^{\circ} \mathrm{C}$ at $10 \mathrm{sec}$, followed by $72^{\circ} \mathrm{C}$ for $2 \mathrm{~min}$ and $16^{\circ} \mathrm{C}$ for $5 \mathrm{~min}$. The reaction mixture contained $2 \mathrm{X}$ UltraSYBR Mixture $(10 \mu \mathrm{l})$, primers (forward/reverse, $0.5 \mu \mathrm{l} / 0.5 \mu \mathrm{l}$ ) and $\mathrm{ddH}_{2} \mathrm{O}(9 \mu \mathrm{l})$.

Luciferase activity assay. For the luciferase activity assay, G401 cells were cultured in a 24 -well plate at a density of $2.5 \times 10^{5}$ cells/well 1 day prior to the transfection. When cells reached $60 \%$ confluence $200 \mathrm{ng}$ pcDNA3.1-luciferase, pcDNA3.1-luciferase-WT1-3'UTR or pcDNA3.1-luciferase-WT1-3'UTR mutant and miR590 mimic or miRNA control plus $10 \mathrm{ng}$ pRLSV40 was used for transfection using Lipofectamine 2000 according to the manufacturer's protocol. The medium was changed after $6 \mathrm{~h}$. Luciferase activity was quantified $36 \mathrm{~h}$ after transfection using Dual Glow Luciferase Assay system (Promega Corporation, Madison, WI, USA). Firefly luciferase activity was normalized to Renilla luciferase activity for each well. Three independent experiments were performed for each group.

5-ethynyl-20-deoxyuridine (EdU) assays. Briefly, G401 cells were seeded into a 24 -well plate at a density of $5 \times 10^{6}$ cells/well 1 day prior to transfection. After $12 \mathrm{~h}, \mathrm{G} 401$ cells were transfected with $10 \mathrm{nM}$ miR-590 mimic, $10 \mathrm{nM}$ miRNA control, $10 \mathrm{nM}$ miR-590 inhibitor, $10 \mathrm{nM}$ miRNA inhibitor, $10 \mathrm{nM}$ si-RNA-WT1, $10 \mathrm{nM}$ si-RNA-control or $800 \mathrm{ng}$ pCDNA3.1-WT1. The proliferation of G401 cells was quantified in vitro $24 \mathrm{~h}$ after transfection by EdU DNA Proliferation and Detection kit (Ribobio Co., Ltd.) following the manufacturer's protocol.

Western blotting. G401 cells were washed with $800 \mu 11 \mathrm{X}$ PBS 3 times for 5 min and lysed with $150 \mu 1$ radioimmunoprecipitation assay per well. For kidney samples, tissue was homogenized in lysis buffer and sonicated for $3 \mathrm{~min}$. The protein concentration of each sample was determined by bicinchoninic acid assay (Beyotime Institute of Biotechnology, Haimen, China). Proteins (50 $\mu \mathrm{g} / \mathrm{lane})$ were 
segregated using 10\% SDS-PAGE gel and immunoblotting was performed using polyclonal antibodies against WT1 (AB10840; 1:2,500; Abcam, Cambridge, UK) and $\beta$-tubulin (AB6040; 1:5,000; Abcam). The two antibodies were used at $4^{\circ} \mathrm{C}$ overnight at $1 \mathrm{mg} / \mathrm{ml}$ in PBS with $5 \%$ non-fat milk according to the manufacturer's protocol. Then the PVDF membrane was probed with horseradish peroxidase (HRP)-conjugated antibodies (1:4,000; Santa Cruz Biotechnology, Inc., Dallas, TX, USA). Finally, immunoreactivity was detected using Chemiluminescent HRP Substrate reagent (Merck Millipore, Darmstadt, Germany) and the signal was analyzed using Bio-Rad ChemiDoc XRS+ (BioRad Laboratories, Inc., Hercules, CA, USA). $\beta$-tubulin was used as an internal control. Western blot analysis was performed in triplicate for each group.

Statistical analysis. Data are expressed as the mean \pm standard deviation. A one-way analysis of variance followed by Newman-Keuls comparison post-hoc test was performed using GraphPad Prism, version 5.0 (GraphPad Software, San Diego, CA, USA). $\mathrm{P}<0.05$ was considered to indicate a statistically significant difference. All of the experimental values are the means of triplicate independent repeats.

\section{Results}

miR-590 expression in nephroblastoma. The present study quantified the expression levels of miR-590 in Wilms' tumor to confirm the involvement of miR-590 in nephroblastoma using RT-qPCR. miR-590 expression levels were significantly higher in Wilms' tumor tissues compared with normal kidney tissue $(\mathrm{P}<0.05$; Fig. 1A). Additionally, the clinical and pathological data of 65 patients with cancer, 25 at stage I/II, 20 at stage III and 20 at stage IV were also evaluated. The expression level of miR-590 was quantified in 65 matched tumor samples compared with the adjacent normal tissues using RT-qPCR. The present study determined that miR-590 was upregulated in 65 tumor tissues compared with the paired adjacent normal tissues. miR-590 expression levels were greater in cancer tissues of patients with stage III or IV tumors compared with stage I/II (Fig. 1B). Therefore, the present study determined that miR-590 upregulation was associated with the development of cancer.

Prediction of miR-590 binding site in the 3'-UTR of WTI $m R N A$. In order to identify the potential downstream targets of miR-590, three independent online databases were used, TargetScan, PicTar and miRBase. Several putative targets were unanimously forecasted using the aforementioned three databases and WT1 was selected as the candidate gene as previous studies have reported its important role in cancer (12-14) and it carries a putative miR-590 binding site within its 3'-UTR, which is located at 1155-1162 bp (Fig. 2A).

miR-590 regulates WT1 expression by binding to its 3'-UTR. In order to verify that miR-590 binds to the predicted region and the binding leads to translational inhibition, a luciferase reporter plasmid with either wide-type or mutant sequence of WT1 mRNA 3'-UTR was cotransfected with miR-590 mimic or miR-control mimic. Successful overexpression of exogenous
miR-590 in G401 cells for 36 h downregulated the activity of the luciferase reporter, which was inserted with wild-type WT1 3'-UTR, whereas the activity with mutated WT1 was not affected, indicating that the mutant target was a potential functional site for miR-590 in WT1 3'-UTR (Fig. 2B and C). In order to determine whether miR-590 has a functional role in WT1 downregulation, G401 cells were treated with miR-590 mimic and western blot analysis indicated that miR-590 overexpression led to a reduction in WT1 protein levels (Fig. 2D). These findings indicated that miR-590-mediated suppression of WT1 expression via binding to 3'-UTR of WT1.

miR-590 promotes cell proliferation. As miR-590 expression was upregulated in Wilms' tumor, which indicated its potential role in the pathogenesis of Wilms' tumor. The effect of increased miR-590 expression on cell proliferation was evaluated. In order to investigate this, miR-590 mimics were transfected into the G401 cells and the effect of miR-590 expression on cell proliferation was analyzed using EdU assay. miR-590 expression level was markedly upregulated in G401 cells treated with miR-590 mimics compared with control mimics, as determined by RT-qPCR ( $\mathrm{P}<0.01 ; 100$-fold). Additionally, cells labeled with EdU were immunostained for EdU (Fig. 3, red), whereas the nuclei were stained with DAPI (Fig. 3, blue). The EdU assay data demonstrated that the overexpression of miR-590 promoted cell proliferation $48 \mathrm{~h}$ after transfection $(\mathrm{P}<0.05$; Fig. 3).

WT1 siRNA transfection leads to G401 cell proliferation. The aforementioned results indicated the potential role of miR-590 in the regulation of G401 cell proliferation and WT1 gene expression. Therefore, the importance of WT1 as a functional target of miR-590 was determined in G401 cell proliferation. To determine the effect of WT1 on cell proliferation, siRNA was used to downregulate WT1 expression levels. WT1 si-RNA markedly reduced WT1 protein expression levels in G401 cells compared with the control siRNA. Following downregulation of WT1 expression levels, a marked increase in proliferation occurred $48 \mathrm{~h}$ after treatment with siRNA. Additionally, the statistical analysis indicated that the number of cells was markedly different between two groups (Fig. 4). These findings suggested that WT1 affected the proliferation in G401 cells.

Antiproliferative effect of WT1 on G401 cells in vitro. The present study determined that miR-590 expression was upregulated in Wilms' tumor, was associated with Wilms' tumor clinical stage, and that miR-590 directly suppressed the expression of WT1. Therefore, the current study aimed to determine whether an increase in WT1 expression may provide an explanation for the observed effects of miR-590 overexpression. WT1 overexpression with pCDNA3.1-WT1 transfection was performed, followed by EdU assay. The successful overexpression of WT1 in G401 cells was confirmed by western blot analysis (Fig. 5). The effect of WT1 treatment for $48 \mathrm{~h}$ on cell proliferation is presented in Fig. 6. Exposure to WT1 led to a reduction in cell proliferation compared with the control group. Therefore, WT1 inhibition may partly elucidate the proliferative effects of miR-590 on G401 cells and WT1 may be involved in the miR-590-mediated proliferation in G401 cells. 
A

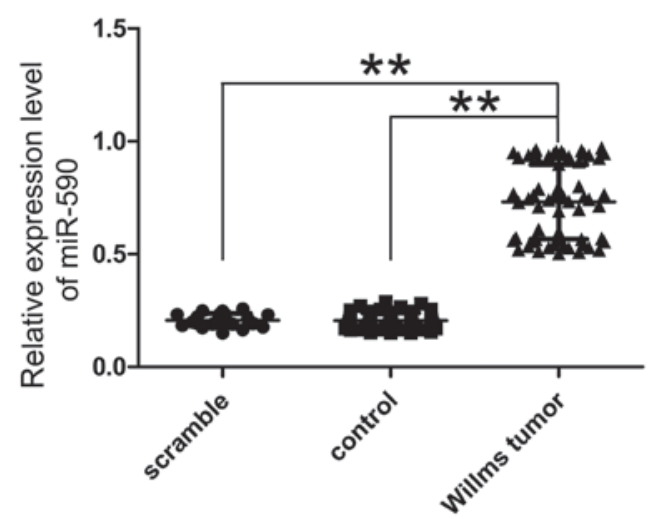

B

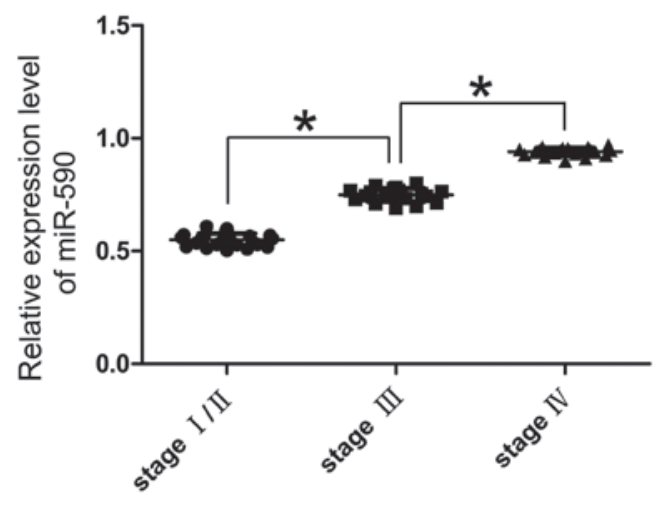

Figure 1. miR-590 was upregulated in Wilms' tumor tissue. (A) Quantitative polymerase chain reaction analysis of miR-590 expression levels in Wilms tumor tissue $(\mathrm{n}=65)$, the adjacent normal tissue $(\mathrm{n}=65)$ and normal kidney $(\mathrm{n}=20)$. The relative quantity of miR-590 was normalized to U6. (B) Expression levels of miR-590 in different clinical tumor stages of Wilms' tumor. The relative quantity of miR-590 was normalized to U6. All data are presented as the mean \pm standard deviation from three independent experiments. ${ }^{*} \mathrm{P}<0.05,{ }^{* *} \mathrm{P}<0.01$. miR, micro RNA.

A

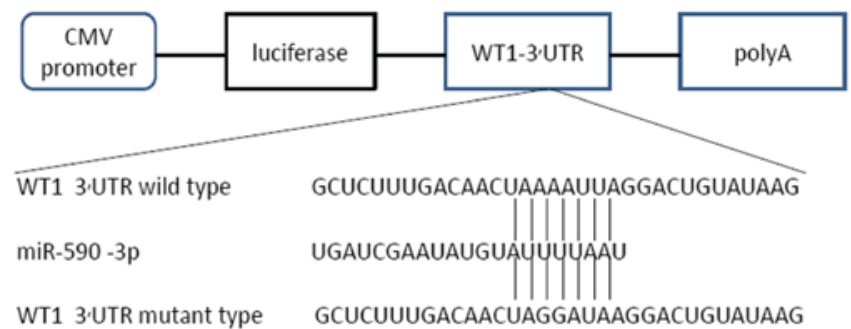

C

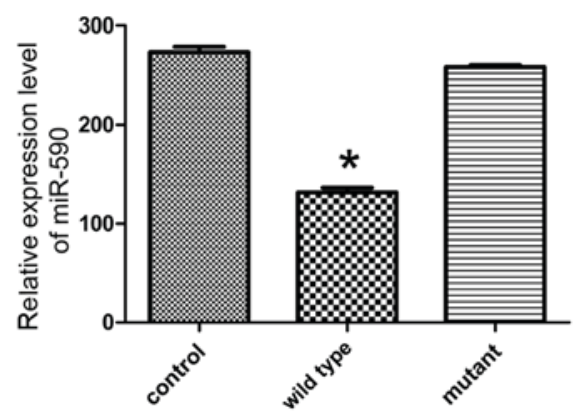

B

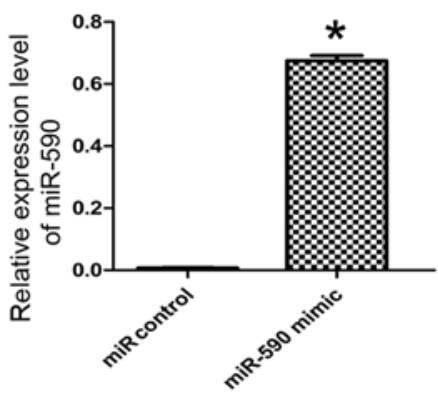

D

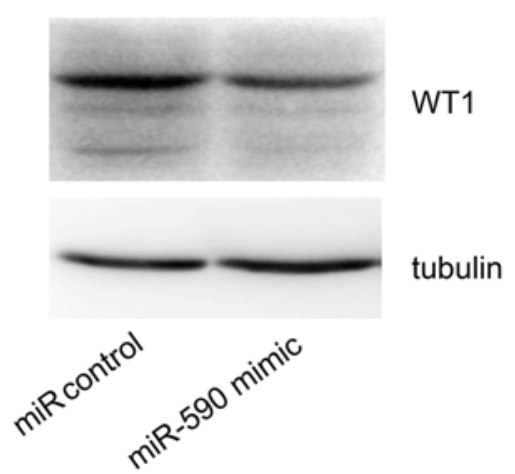

Figure 2. miR-590 targeted WT1 and its expression. (A) Schematic presentation of the binding site of miR-590 in the 3'-UTR of WT1 and WT1 3'-UTR luciferase reporter constructs, including wild-type and mutant type. (B) Validation of the transfection of miR-590 by using reverse transcription-quantitative polymerase chain reaction. (C) Dual-luciferase reporter assays indicated that in the wild-type miR-590 expression was reduced, and in the mutant, the WT1 3'-UTR binding site was affected, thus activity in G401 cells was inhibited following transfection with miR-590 mimic. (D) Western blot analysis of WT1 expression level in cultured G401 cells transfected with miR-590 (10 nM) or miR-NC. Quantitative analysis of WT1 protein level was normalized to $\beta$-tubulin. All data are presented as the mean \pm standard deviation from three independent experiments. ${ }^{*} \mathrm{P}<0.01 \mathrm{vs.} \mathrm{miR-control.} \mathrm{CMV}$, cytomegalovirus; WT1, Wilms' tumor 1; 3'-UTR, 3'-untranslated region; miR, microRNA.

\section{Discussion}

Previous studies have identified that miRNAs are key in novel pathways of carcinogenesis $(4,5,15)$. miRNAs function either as tumor suppressors or oncogenes. Aberrant expression of miR-590 has been identified in various types of human cancers, including acute myeloid leukemia (16), hepatocellular carcinoma (6), cervical cancer (10) and kidney cancer (7). Additionally, previous studies have suggested that miR-590 upregulation may be associated with cancer metastasis $(6,7)$. A previous study demonstrated that the miR-590 expression level was significantly higher in recurrent compared with primary tumors in GC cells and tissues (17). Therefore, miR-590 may act as a tumor promoter in some malignant diseases; however, in order to elucidate the specific functions, further investigation is required.

The present study demonstrated that the endogenous miR-590 expression level was significantly upregulated in 

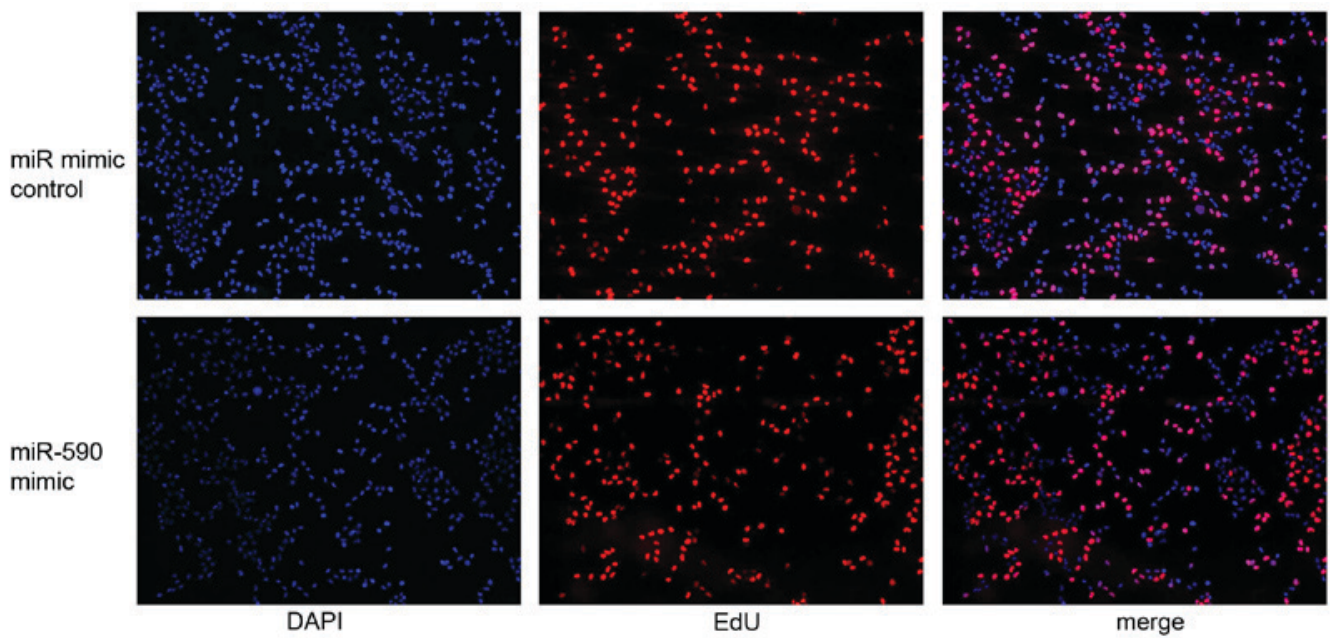

Figure 3. miR-590 affects G401 cell proliferation. G401 morphology was determined by fluorescence microscopy. Proliferation was assessed using an EdU cell proliferation assay kit. G401 cells were stained by EdU and DAPI $36 \mathrm{~h}$ after transfection. Cell proliferation rate was increased following the overexpression of miR590. Magnification, x100. miR, micro RNA; EdU, 5-ethynyl-20-deoxyuridine.

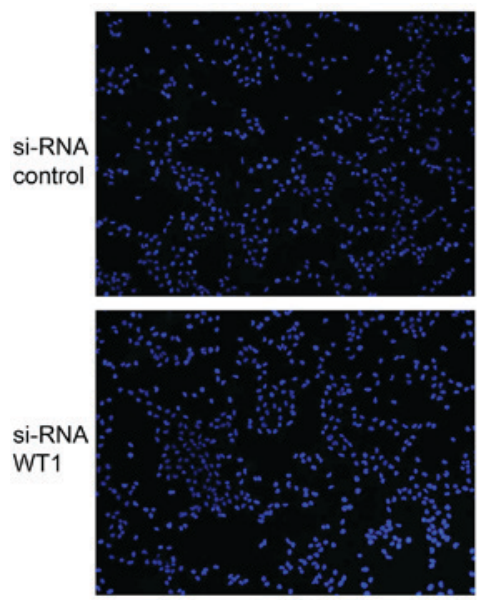

DAPI
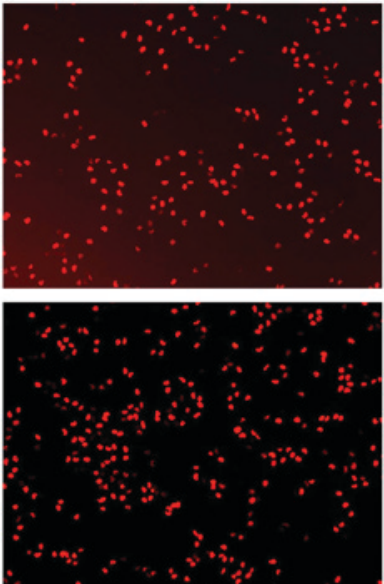

EDU
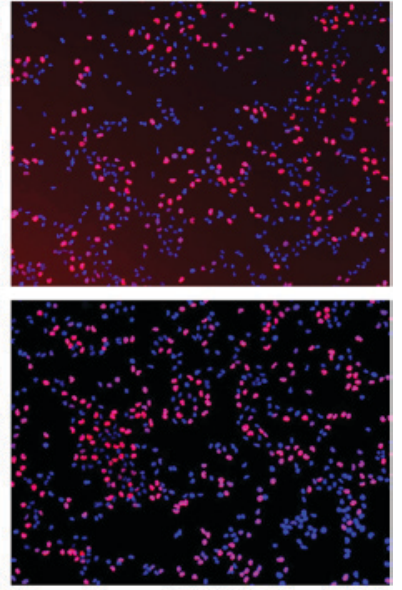

merge

Figure 4. Importance of WT1 in G401 cell proliferation using siRNA. G401 morphology was determined by fluorescence microscopy. Proliferation was assessed using an EdU cell proliferation assay kit. G401 cells were stained by EdU and DAPI $36 \mathrm{~h}$ after transfection. Cell proliferation rate was increased following overexpression of si-RNA-WT1. Magnification, x100. siRNA, small interfering RNA; WT1, Wilms' tumor 1; EdU, 5-ethynyl-20-deoxyuridine.

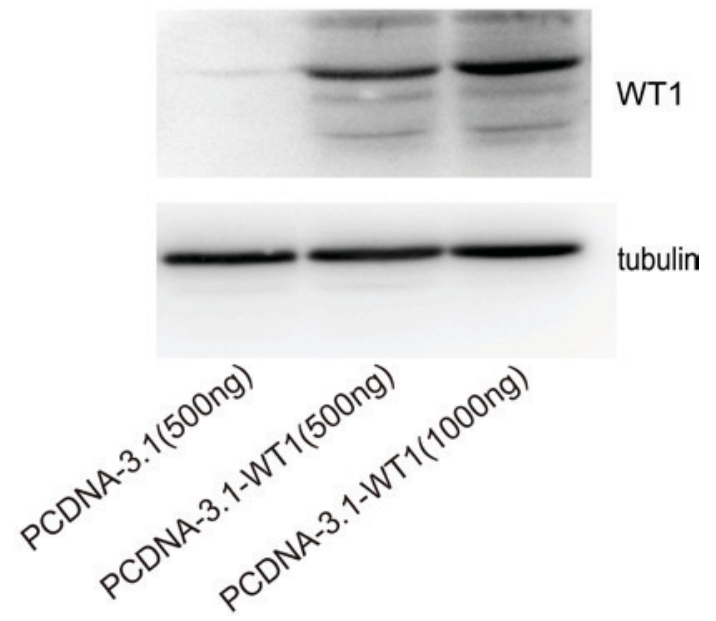

Figure 5. Western blot analysis of WT1 expression level in cultured G401 cells. Quantitative analysis of WT1 protein levels was normalized to $\beta$-tubulin. WT1 was successfully expressed in G401 cells. PCDNA-3.1 and PCDNA-3.1-WT1 plasmids (500 or 1,000 ng) were transfected into G401 cells with Lipofectamine 2000. WT1, Wilms' tumor 1.
Wilms' tumor tissue compared with normal renal tissue, which was consistent with a previous study reporting high miR-590 levels in kidney cancer tissues (7). Subsequently gain-of-function and loss-of-function approaches were used by transfection with miR-590 mimics or miR-590 inhibitor in G401 cells to determine the function of miR-590. Change in the rate of cell proliferation is one of the key phenotypes observed in malignant transformation, which may lead to a human cancer mortality rate as high as $90 \%(18,19)$. The findings of the current study indicated that the overexpression of miR-590 increased G401 cell proliferation, having a tumor promoter role in Wilms' tumor development.

Several biological targets of miR-590 have been identified. For example, miR-590 downregulates PBRM1 expression, leading to increased cell proliferation and invasion in CCRCC (7) and promotes cervical cancer cell growth and invasion by targeting CHL1 (10). The present study demonstrated that miR-590 inhibited WT1 expression by targeting the $3^{\prime}$-UTR, indicating that WT1 is a direct target 

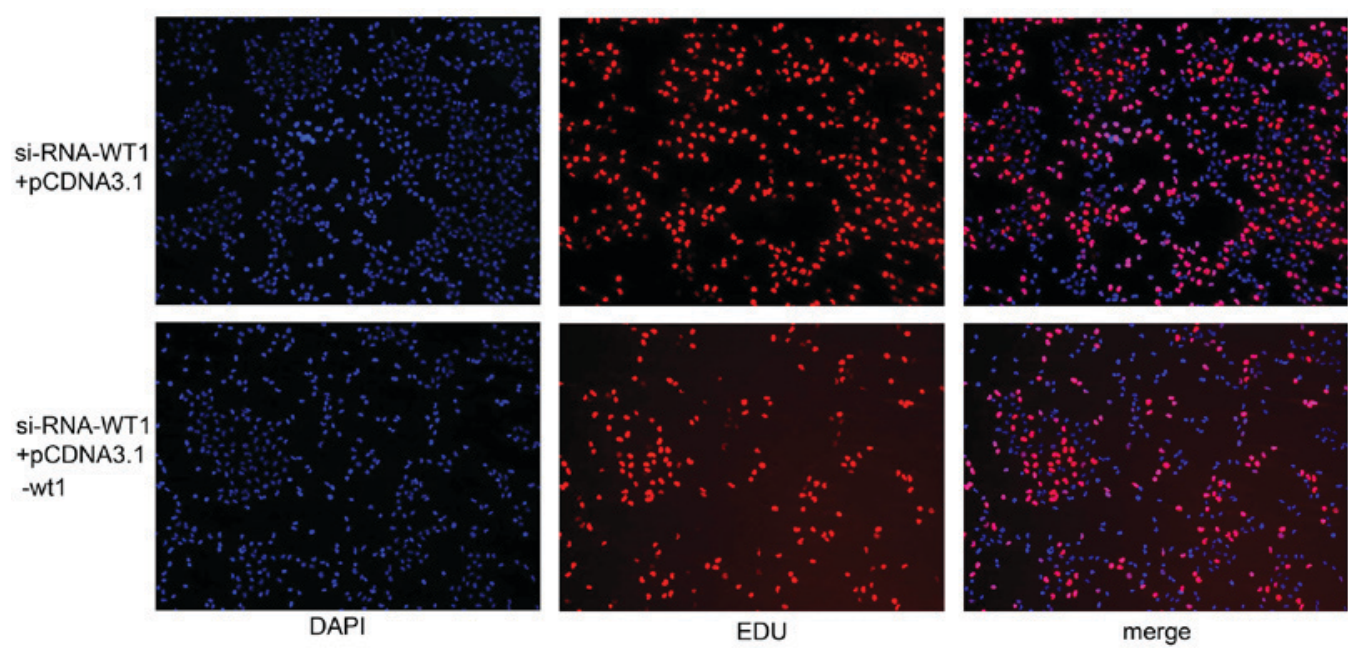

Figure 6. Importance of WT1 in G401 cell proliferation following WT1 overexpression. G401 morphology was determined by fluorescence microscopy. Proliferation was assessed using the EdU cell proliferation assay kit. G401 cells were stained by EdU and DAPI $36 \mathrm{~h}$ after transfection. Cell proliferation rate was decreased following WT1 overexpression. siRNA, small interfering RNA; WT1, Wilms' tumor 1; EdU, 5-ethynyl-20-deoxyuridine.

of miR-590. As the Wilms' tumor transcription factor (WT1) was originally classified as a tumor suppressor, miR-590 may be a tumor activator, inversely associated with its specific target gene.

Wilms' tumor has an incidence of $\sim 1$ in 10,000 live births and is one of the most common solid tumors occurring in childhood in the developed world (20-22). The mean age of patients when diagnosed is 43-48 months, depending on gender (women express clearer clinical symptoms thus are likely to be diagnosed earlier) and $95 \%$ of patients are diagnosed by 10 years of age $(20,21)$. Tumors often occur unilaterally in $90-95 \%$ of cases (in a single kidney); however, they may also occur bilaterally. The bilateral patients present clinical symptoms 12 months earlier compared with unilateral patients (22-24). According to previous studies, $\sim 2 \%$ of cases have an affected relative and Wilms' tumor predisposition segregates in families as an autosomal dominant trait $(23,24)$. The WT1 tumor suppressor factors, which were involved in the development of nephroblastoma, were the first tumor repressor genes to be cloned $(25,26)$. Observational data from Wilms' tumors and acute myeloid leukemia combined with improved knowledge of temporal and spatial expression levels of WT1 in nephrogenesis and hematopoiesis suggest that functional loss of WT1 has an important role in a subset of these malignant diseases (27-30). However, in vivo and in vitro experimental data indicated that functional loss of WT1 may result in differing phenotypic consequences, including apoptosis, quiescence or proliferation, depending on the differentiation status of the cell (31-33). In the present study, the knockdown of WT1 expression by siRNA-WT1 had a proliferative effect on G401 cells, similar effect to overexpression of miR-590. WT1 upregulation may rescue the cell proliferative phenotype of G401 cells via siRNA-WT1 (34). Therefore, WT1 may be one of the key mediators of G401 suppression by miR-590. A previous study suggested that WT1 may be involved in the progression of cancer (29). As WT1 is a target gene of miR-590, the suppression of WT1 expression may be due to miR-590 overexpression in Wilms tumor.
In conclusion, the results of the present study are consistent with the hypothesis that miR-590 may promote G401 cell proliferation via downregulation of it specific target gene, WT1 as miR-590 expression level increased in Wilms' tumor tissues compared with normal kidney tissues. A direct and functional target of miR-590, WT1, is downregulated and associated with cell proliferation in G401. Further investigations should be undertaken and directed toward complete understanding of the underlying molecular mechanism of the miRNA upregulation within tumorigenesis, in order to develop promising strategies for targeted therapy of Wilms' tumor in the future.

\section{Acknowledgements}

The present study was supported by a grant from the Science and Technology Program in Suzhou (grant no. sys201434) to Professor Hong Zhu.

\section{References}

1. Tournade MF, Com-Nougué C, Voûte PA, Lemerle J, de Kraker J, Delemarre JF, Burgers M, Habrand JL, Moorman CG, Bürger D, et al: Results of the sixth international society of pediatric oncology Wilms' tumor trial and study: A risk-adapted therapeutic approach in Wilms' tumor. J Clin Oncol 11: 1014-1023, 1993.

2. Graf N, van Tinteren H, Bergeron C, Pein F, van den Heuvel-Eibrink MM, Sandstedt B, Schenk JP, Godzinski J, Oldenburger F, Furtwängler R and de Kraker J: Characteristics and outcome of stage II and III non-anaplastic Wilms' tumour treated according to the SIOP trial and study 93-01. Eur J Cancer 48: 3240-3248, 2012.

3. Davidoff AM, Giel DW, Jones DP, Jenkins JJ, Krasin MJ Hoffer FA, Williams MA and Dome JS: The feasibility and outcome of nephron-sparing surgery for children with bilateral Wilms' tumor. The St Jude children's research hospital experience: 1999-2006. Cancer 112: 2060-2070. 2008.

4. He L and Hannon GJ: MicroRNAs: Small RNAs with a big role in gene regulation. Nat Rev Genet 5: 522-531, 2004.

5. Akkina $\mathrm{S}$ and Becker BN: MicroRNAs in kidney function and disease. Transl Res 157: 236-240, 2011.

6. Jiang X, Xiang G, Wang Y, Zhang L, Yang X, Cao L, Peng H, Xue $P$ and Chen D: MicroRNA-590-5p regulates proliferation and invasion in human hepatocellular carcinoma cells by targeting TGF- $\beta$ RII. Mol Cells 33: 545-551, 2012. 
7. Xiao X, Tang C, Xiao S, Fu C and Yu P: Enhancement of proliferation and invasion by MicroRNA-590-5p via targeting PBRM1 in clear cell renal carcinoma cells. Oncol Res 20: 537-544. 2013

8. Shan X, Miao Y, Fan R, Qian H, Chen P, Liu H, Yan X, Li J and Zhou F: MiR-590-5P inhibits growth of HepG2 cells via decrease of S100A10 expression and inhibition of the Wnt pathway. Int J Mol Sci 14: 8556-8569, 2013.

9. Liu T, Nie F, Yang X, Wang X, Yuan Y, Lv Z, Zhou L, Peng R, $\mathrm{Ni} \mathrm{D}, \mathrm{Gu} \mathrm{Y}$, et al: MicroRNA-590 is an EMT-suppressive microRNA involved in the TGF- $\beta$ signaling pathway. Mol Med Rep 12: 7403-7411, 2015.

10. Chu Y, Ouyang Y, Wang F, Zheng A, Bai L, Han L, Chen Y and Wang H: MicroRNA-590 promotes cervical cancer cell growth and invasion by targeting CHL1. J Cell Biochem 115: 847-853, 2014.

11. Tongaonkar HB, Qureshi SS, Kurkure PA, Muckaden MA, Arora B, Yuvaraja TB: Wilms Tumor: An Update. Indian J Urol 23: 458-466, 2007.

12. Menssen HD, Siehl JM and Thiel E: Wilms tumor gene (WT1) expression as a panleukemic marker. Int J Hematol 76: 103-109, 2002.

13. Wang X, Gao P, Lin F, Long M, Weng Y, Ouyang Y, Liu L, Wei J, Chen X, He T, et al: Wilms' tumour suppressor gene 1 (WT1) is involved in the carcinogenesis of Lung cancer through interaction with PI3K/Akt pathway. Cancer Cell Intern 13: 114, 2013.

14. Tatsumi N, Oji Y, Tsuji N, Tsuda A, Higashio M, Aoyagi S, Fukuda I, Ito K, Nakamura J, Takashima S, et al: Wilms' tumor gene WT1-shRNA as a potent apoptosis-inducing agent for solid tumors. Int J Oncol 32: 701-711, 2008.

15. Zhang $\mathrm{J}$ and Ma L: MicroRNA control of epithelial-mesenchymal transition and metastasis. Cancer Metast Rev 31: 653-662, 2012

16. Favreau AJ and Sathyanarayana P: miR-590-5p, miR-219-5p, miR-15b and miR-628-5p are commonly regulated by IL-3, GM-CSF and G-CSF in acute myeloid leukemia. Leuk Res 36: 334-341, 2012.

17. Jalava SE, Urbanucci A, Latonen L, Waltering KK, Sahu B, Jänne OA, Seppälä J, Lähdesmäki H, Tammela TL and Visakorpi T: Androgen-regulated miR-32 targets BTG2 and is overexpressed in castration-resistant prostate cancer. Oncogene 31: 4460-4471, 2012.

18. Yifru S and Muluye D: Childhood cancer in Gondar University Hospital, Northwest Ethiopia. BMC Res Notes 8: 474, 2015.

19. Ochicha O, Gwarzo AK and Gwarzo D: Pediatric malignancies in Kano, Northern Nigeria. World J Pediatr 8: 235-239, 2012.

20. Chu A, Heck JE, Ribeiro KB, Brennan P, Boffetta P, Buffler P and Hung RJ: Wilms' tumour: A systematic review of risk factors and meta-analysis. Paediatr Perinat Epidemiol 24: 449-469, 2010

21. Scélo G and Brennan P: The epidemiology of bladder and kidney cancer. Nat Clin Prac Urol 4: 205-217, 2007.
22. Schüz J, Schmidt LS, Kogner P, Lähteenmäki PM, Pal N, Stokland $\mathrm{T}$ and Schmiegelow K: Birth characteristics and Wilms tumors in children in the Nordic countries: A register-based case-control study. Int J Cancer 128: 2166-2173, 2011.

23. Beckwith JB, Kiviat NB and Bonadio JF: Nephrogenic rests, nephroblastomatosis and the pathogenesis of Wilms' tumor. Pediatr Pathol 10: 1-36, 1990.

24. Beckwith JB: Nephrogenic rests and the pathogenesis of Wilms tumor: Developmental and clinical considerations. Am J Med Genet 79: 268-273, 1998.

25. Dallosso AR, Hancock AL, Brown KW, Williams AC, Jackson S and Malik K: Genomic imprinting at the WT1 gene involves a novel coding transcript (AWT1) that shows deregulation in Wilms' tumours. Hum Mol Genet 13: 405-415, 2004.

26. Menke AL, van der Eb AJ and Jochemsen AG: The Wilms' tumor 1 gene: Oncogene or tumor suppressor gene? Int Rev Cytol 181: $151-212,1998$.

27. Amini Nik S, Hohenstein P, Jadidizadeh A, Van Dam K, Bastidas A, Berry RL, Patek CE, Van der Schueren B, Cassiman JJ and Tejpar S: Upregulation of Wilms' tumor gene 1 (WT1) in desmoid tumors. Int J Cancer 114: 202-208, 2005.

28. Oji Y, Suzuki T, Nakano Y, Maruno M, Nakatsuka S, Jomgeow T, Abeno S, Tatsumi N, Yokota A, Aoyagi S, et al: Overexpression of the Wilms' tumor gene W T1 in primary astrocytic tumors. Cancer Sci 95: 822-827, 2004.

29. Oka Y, Tsuboi A, Taguchi T, Osaki T, Kyo T, Nakajima H, Elisseeva OA, Oji Y, Kawakami M, Ikegame K, et al: Induction of WT1 (Wilms' tumor gene)-specific cytotoxic T lymphocytes by WT1 peptide vaccine and the resultant cancer regression. Proc Natl Acad Sci USA 101: 13885-13890, 2004.

30. Yang L, Han Y, Suarez Saiz F and Minden MD: A tumor suppressor and oncogene: The WT1 story. Leukemia 21: 868-876, 2007.

31. Hohenstein P and Hastie ND: The many facets of the Wilms' tumour gene, WT1. Hum Mol Genet 15 Spec No 2: R196-R201, 2006.

32. Xu C, Wu C, Xia Y, Zhong Z, Liu X, Xu J, Cui F, Chen B, Røe OD, Li A and Chen Y: WT1 promotes cell proliferation in non-small cell lung cancer cell lines through up-regulating cyclin D1 and p-pRb in vitro and in vivo. PLoS One 8: e68837, 2013.

33. Algar EM, Khromykh T, Smith SI, Blackburn DM, Bryson GJ and Smith PJ: A WT1 antisense oligonucleotide inhibits proliferation and induces apoptosis in myeloid leukaemia cell lines. Oncogene 12: 1005-1014, 1996.

34. Davies JA, Ladomery M, Hohenstein P, Michael L, Shafe A, Spraggon L and Hastie N: Development of an siRNA-based method for repressing specific genes in renal organ culture and its use to show that the $\mathrm{Wt1}$ tumour suppressor is required for nephron differentiation. Hum Mol Genet 13: 235-246, 2004. 